\title{
Unsteady three-dimensional flow and heat transfer past a permeable stretching/shrinking surface
}

\begin{abstract}
The unsteady laminar three-dimensional boundary layer viscous flow and heat transfer over a permeable stretching/shrinking surface (sheet) is studied numerically. The system of nonlinear partial differential equations is transformed into ordinary differential equations via the similarity transformation. The resulting governing equations are then solved numerically by using the shooting method. Effects of the unsteadiness parameter, the stretching/shrinking parameter, the mass suction parameter and the Prandtl number on the skin friction coefficients, the local Nusselt number as well as the velocity and temperature profiles are presented and discussed in detail. The results also show that dual solutions exist for both cases of the stretching and shrinking surfaces.
\end{abstract}

Keyword: Boundary layer; Heat transfer; Stretching/shrinking surface; Unsteady threedimensional flow 